
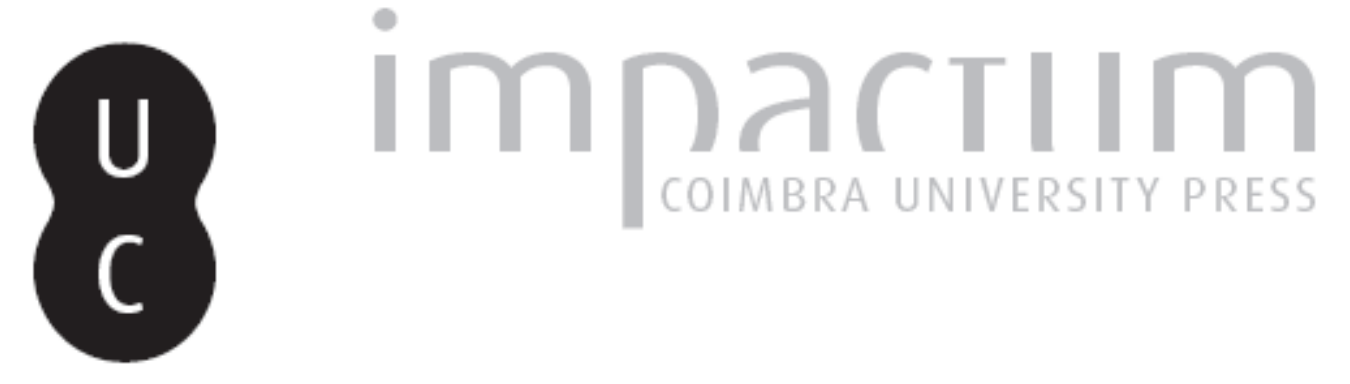

\title{
Burdigala et l'organization de la province romaine d'Aquitaine
}

\author{
Autor(es): Le Roux, Patrick
}

Publicado por: Faculdade de Letras da Universidade de Coimbra

URL persistente:

URI:http://hdl.handle.net/10316.2/37862

DOI:

DOI:http://dx.doi.org/10.14195/1647-8657_49_6

Accessed : $\quad$ 26-Apr-2023 05:35:49

A navegação consulta e descarregamento dos títulos inseridos nas Bibliotecas Digitais UC Digitalis, UC Pombalina e UC Impactum, pressupõem a aceitação plena e sem reservas dos Termos e Condições de Uso destas Bibliotecas Digitais, disponíveis em https://digitalis.uc.pt/pt-pt/termos.

Conforme exposto nos referidos Termos e Condições de Uso, o descarregamento de títulos de acesso restrito requer uma licença válida de autorização devendo o utilizador aceder ao(s) documento(s) a partir de um endereço de IP da instituição detentora da supramencionada licença.

Ao utilizador é apenas permitido o descarregamento para uso pessoal, pelo que o emprego do(s) título(s) descarregado(s) para outro fim, designadamente comercial, carece de autorização do respetivo autor ou editor da obra.

Na medida em que todas as obras da UC Digitalis se encontram protegidas pelo Código do Direito de Autor e Direitos Conexos e demais legislação aplicável, toda a cópia, parcial ou total, deste documento, nos casos em que é legalmente admitida, deverá conter ou fazer-se acompanhar por este aviso.

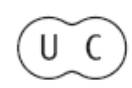


CONIMBRIGA

C)

CN N N

INSTITUTO DE ARQUEOLOGIA

VOLUME XLIX • 2010

FACULDADE DE LETRAS 
PATRICK LE ROUX

Professeur émérite. CRESC Paris 13

BURDIGALA ET L'ORGANISATION

DE LA PROVINCE ROMAINE D'AQUITAINE

“Conimbriga" XLIX (2010) p. 97-118

RÉSUMÉ: La question de la capitale de la province romaine d'Aquitaine a donné lieu à des débats anciens, sans issue jusqu'à présent en raison d'une documentation qui l'ignore et de lectures modernes déformantes. Replacée dans le contexte du regard géographique antique et de la construction administrative impériale, étrangère aux critères archéologiques et centralisateurs, l'identification doit écarter un déplacement au cours des siècles qui serait sans exemple. Burdigala fut la sedes ordinaire du gouverneur provincial dès Auguste. Pour d'autres cités l'ambiguïté a pu tenir à la fonction de siège du conventus judiciaire, ce qui ne fut pas non plus le cas de Saintes toutefois.

Resumo: A questão da capital da província romana da Aquitânia deu origem a velhos debates, sem solução até agora, devido a uma documentação que a ignora e de leituras actuais distorcidas. Vista no contexto da visão geográfica antiga e da construção administrativa imperial, estranha aos critérios arqueológicos e centralizadores, a identificação deve excluir, por falta de paralelos, a hipótese de ter havido, a certa altura, uma transferência. Burdigala foi a sedes ordinária do governador provincial desde Augusto. Para outras cidades, a ambiguidade pode explicar-se pelo facto de terem sido sedes de conventus judiciais - o que não foi, todavia, o caso de Saintes.

Conimbriga, 49 (2010) 97-118 
(Página deixada propositadamente em branco) 


\section{BURDIGALA ET L'ORGANISATION DE LA PROVINCE ROMAINE D'AQUITAINE}

Il n'y a aucun document bien connu ou de découverte relativement récente qui nous informe directement sur l'organisation administrative des provinces d'Aquitaine au Haut-Empire romain ${ }^{1}$. Les questions débattues sont toujours celles que dut affronter Robert Étienne, historien de Burdigala et spécialiste de l'Aquitaine d'époque antique ${ }^{2}$. Parmi elles, l'identification de la capitale provinciale ne laisse pas de faire problème depuis le $\mathrm{XIX}^{\mathrm{e}}$ siècle, ce qui étonne et devrait étonner. L'affaire tourne autour de Bordeaux et Saintes mais Poitiers a aussi ses partisans ${ }^{3}$. Devant un tel constat, il paraît logique de conclure que les modes de raisonnement utilisés jusqu'à présent n'ont pas débouché sur des résultats entièrement fiables ${ }^{4}$. La grille de lecture, comme presque toujours, tient une place centrale et ce quelle que soit l'approche adoptée. Robert Étienne, attaché passionnément à l'intelligence historique des faits, refusait les cloisonnements le plus souvent appauvrissants entre les disciplines. Dans cette direction, il convient de réfléchir aux présupposés implicites propres à toute forme d'interprétation sachant que les faits sont

1 Le statut impérial prétorien de la province qui n'est pas discutable n'est évidemment pas concerné ; il est illustré en particulier par la carrière d'Agricola (infra n. 45).

2 Bordeaux antique, 1962, En passant par l'Aquitaine..., 1995, etc. Certains débats concernant la lecture des textes tendaient, il est vrai, à monopoliser les recherches. Robert Étienne entendait y prendre toute sa part après avoir fondé en érudition et en réflexion ce qu'il appelait sa « doctrine ».

3 Par exemple Tranoy, 1984, p. 283. Voir en dernier lieu HaEnsch, 1997, TRAnoy, 2004 et les réflexions en forme de bilan de Maurin, 2005.

4 HAENSCH, 1997, propose une critique systématique des arguments accumulés par leur confrontation avec les sources mais il ne risque pas de conclusion ferme et argumentée aux débats.

Conimbriga, 49 (2010) 97-118 
toujours construits et ne parlent pas seuls, sans intervention extérieure. L'imagination est nécessaire ; elle demeure une alliée capricieuse et difficile lorsqu'elle a tendance à prendre trop de place.

Les données méthodologiques sont moins simples qu'il n'y paraissait de prime abord. Dans quelle mesure peut-on demander aux vestiges archéologiques d'éclairer des problèmes d'histoire politique et administrative, sachant que le puzzle conservé est fragmentaire et implique d'y placer des pièces isolées dont rien ne dit qu'elles en font partie ? Ce n'est qu'après avoir débrouillé les fils emmêlés par la superposition d'arguments disparates qu'il sera possible de recourir à de nouvelles propositions mieux justifiées et mieux ciblées.

\section{Où les documents ne disent rien}

Une observation s'impose au départ. En dehors de la Notice des Gaules désignant Burdigala comme metropolis de l'Aquitaine Seconde ${ }^{5}$, il n'y a pas dans les textes d'indication disponible relative au statut administratif des villes et cités de la province augustéenne et postérieure. Strabon, le premier chronologiquement, n'offre aucun secours ni point d'ancrage ${ }^{6}$. Il ressort essentiellement de sa description sommaire que les Santons étaient limitrophes de la Garonne au nord et faisaient face aux Bituriges Vivisques intégrés à l'Aquitaine césarienne

5 Not. Gall., XIII, 1, datable vers 400 p. C. Le terme de metropolis utilisé dans ce document est à lire dans un sens dérivé et tardif pour la résidence du gouverneur et comporte sans doute désormais une dimension honorifique que n'avait pas le caput au Haut-Empire. L'Aquitaine Seconde correspondait à la moitié occidentale de la nouvelle Aquitaine augustéenne agrandie de la cité des Bituriges Vivisques.

6 Les extraits concernés sont bien connus et souvent commentés ce qui ne veut pas dire que toutes les difficultés en sont résolues unanimement : (IV, 2, 1) « $L a$ Garonne... a son embouchure entre le pays des Bituriges dits Bituriges Vivisques et celui des Santons, peuples gaulois tous les deux. De fait, ces Bituriges sont la seule population allogène établie chez les Aquitains et ils ne paient pas l'impôt avec eux; ils ont pour place de commerce Burdigalla au bord d'une lagune formée par l'estuaire de la Garonne... La ville chef-lieu des Santons est Mediolanium. » L'expression négative ou sunteleî autoîs concernant les habitants de Burdigala ne peut pas signifier autre chose qu'une exemption des contributions versées à Rome par les Aquitains (la traduction de Desjardins était déjà la bonne en soulignant la non association avec les Aquitains, malgré BATS, 2007, p. 58) à l'époque d'Auguste ce qui trouve une explication chez PLINE (infra) qui parle de cité libre (NH, IV, 108).

Conimbriga, 49 (2010) 97-118 
au sud du fleuve. En dehors de la renommée somme toute banale de l'un et l'autre peuple parmi les plus importants de ces régions, il n'est question d'aucun statut particulier. Dans le même passage, il est noté que les Namnètes sont séparés des Pictons par la Loire suivant une présentation géographique qui ignore l'administration. Lorsque Strabon avance des informations autres, il est toujours précis comme l'atteste la remarque sur les Rèmes dont la ville Duricortora, jugée la plus peuplée des villes de la région, est dite résidence des gouverneurs (hêgemones) ${ }^{7}$. À propos de Lugdunum $^{8}$, propriété des Romains, Strabon indique aussi qu'elle est la plus peuplée après Narbonne et que les représentants de Rome (hêgemones) y battent monnaie d'or et d'argent. En dehors de quelques observations d'actualité reflétant la mise à jour du texte par endroits, Strabon n'est que peu soucieux des statuts et des détails administratifs, sauf si ceux-ci renforcent l'orientation d'un propos tourné vers l'euandria', l'ancienneté, la renommée ou l'importance présente d'une communauté ou d'une région. S'agissant de fixer la capitale de l'Aquitaine augustéenne et de choisir entre les Santons et les Vivisques ou les Pictons/Pictaves, malgré ce qui est dit souvent, le géographe d'Amasée n'est d'aucun secours. Sa démarche d'ensemble ou de détail ne tient pas compte du découpage provincial augustéen.

Pline l'Ancien n'ignore pas les circonscriptions provinciales des Romains mais il n'en est pas l'esclave. Il s'en tient souvent à une sèche énumération et cite en l'occurrence dans l'ordre «les Pictons, les Santons libres, les Bituriges libres surnommés Vivisques, les Aquitains qui donnent son nom à la province...». Le statut de cité libre est également celui des Bituriges Cubes et des Arvernes. La liberté de ces peuples dont l'origine césarienne ou augustéenne n'est pas rapportée dans les sources paraît être une reconnaissance d'une puissance ancienne et redoutée ou méritante. La réunion dans une liste des trois cités candidates au rang de capitale provinciale suit un itinéraire odographique du nord au sud et ne suggère rien d'autre. Comme Strabon qu'il ne cite ni n'utilise, Pline n'est pas soucieux de désigner les capita provinciarum sans doute parce que ses sources ne tenaient pas compte de cette donnée et n'avaient aucune

\footnotetext{
IV, 3, 5. La forme latine équivalente est praeses.

$8 \mathrm{IV}, 3,2$.

9 La densité démographique est un critère de prospérité et un gage d'avenir.
}

Conimbriga, 49 (2010) 97-118 
raison de le faire ${ }^{10}$. Tacite parlant de son beau-père Agricola rappelle qu'il fut nommé par Vespasien au gouvernement de l'Aquitaine, spes consulatus, ce qu'il ne faut sans doute pas généraliser mais interpréter à cette époque essentiellement en fonction de précédents et de la carrière d'Agricola $^{11}$. Il n'est fait aucune allusion à la résidence du légat prétorien impérial bien connue ou sans signification particulière.

Plus éloquent en apparence est le témoignage d'Eutrope : Tetricus senator qui Aquitaniam honore praesidis administrans absens a militibus imperator electus est et apud Burdigalam purpuram sumpsit ${ }^{12}$. Le Bréviaire adressé à Valens est un résumé chronologique destiné à l'édification de l'empereur. Les faits ne sont pas recevables sans examen et l'élection d'un sénateur absent par les soldats souligne une anomalie, dans le contexte politique de l'époque ${ }^{13}$. Il est vrai qu'il s'agit d'un usurpateur aux yeux de l'empereur de Rome, Aurélien, et que le choix d'un sénateur civil pouvait sembler habile en cas de négociations ou de défaite. La version de l'Histoire Auguste met en avant les intrigues de l'impératrice Victoria mère de Victorin dont le meurtre entraîna la désignation de Tetricus ${ }^{14}$. L'épisode rapporté par Eutrope garde sans aucun doute la mémoire de Burdigala comme centre administratif servant de résidence au gouverneur. Ce n'est qu'un indice qui ne peut pas être tenu objectivement pour décisif en tant que tel. Il suffirait de rappeler que Galba était à Carthagène quand il fut sollicité contre Néron et que c'est à Clunia qu'il apprit sa proclamation par le sénat le 10 juin 68 p. C. ${ }^{15}$. Tetricus était un autre Galba aux yeux d'une tradition hostile aux

10 L'usage de « caput» n'est pas exempt d'ambiguïté chez Pline : Rome est décrite comme la tête posée sur le corps que serait l'Italie et le caput Celtiberiae se référant aux Segobrigenses a le même sens concret de ce qui dépasse, est visible et en même temps permet d'ordonner et de faire vivre.

11 TACITE, Vie d'Agricola, IX. Un anachronisme du biographe écrivant à la fin du $\mathrm{I}^{\mathrm{er}}$ s. p. C. n'est pas exclu sur ce point.

12 Eutrope, Bréviaire, IX, 10 : «Le sénateur Tetricus qui administrait l'Aquitaine en qualité de præses fut choisi comme empereur en son absence par les soldats et revêtit la pourpre à Burdigala. »

13 Voir aussi Chastagnol, Histoire Auguste, p. 852. La carrière même de Tetricus est mal connue et suggère que des sénateurs continuaient à gouverner des provinces de l'empereur, du moins celles de rang prétorien.

14 La vie de Tetricus est intégrée aux « Trente Tyrans » dont le contenu est très sujet à caution.

15 Le Roux, 1982, p. 134-135.

Conimbriga, 49 (2010) 97-118 
« mauvais empereurs » mais, comme pour la péninsule Ibérique, les lieux n'ont pas été choisis ni inventés au hasard ${ }^{16}$. Burdigala abritait probablement la résidence du gouverneur d'Aquitaine au $\mathrm{III}^{\mathrm{e}}$ siècle, ce que suggère aussi son rang à la tête de l'Aquitaine Seconde au $\mathrm{IV}^{17}$.

Les inscriptions sont également sollicitées bien qu'elles ne mentionnent jamais le «caput provinciae », expression d'ailleurs pour ainsi dire absente des sources ${ }^{18}$. Limonum Pictonum a produit un document consacré à la femme défunte d'un légat de la province, morte à Poitiers au $\mathrm{II}^{\mathrm{e}}$ siècle probablement ${ }^{19}$. Le fait qu'il est présenté comme leg. Aug. pr. pr. pr. Aquitan. sur une base de statue dédiée par la civitas Pictonum à son épouse décédée incite à penser que Limonum n'était pas le lieu habituel de résidence du légat. L'hommage est exceptionnel parce que la cité s'honore de pouvoir indirectement marquer sa déférence envers un personnage puissant qu'elle n'avait sans doute pas eu l'occasion de célébrer autrement. Comme le rappelle en outre, parmi d'autres témoignages dans l'Empire, l'inscription fragmentaire de Limoges, il n'était pas nécessaire d'être une capitale provinciale pour décider de faire

16 Il n'est toutefois pas indifférent que ce soit Burdigala et non Saintes ou Poitiers qui est nommée. Voir aussi infra n. 47 : Galba avait gouverné l'Aquitaine au cours de sa carrière juste avant son consulat.

17 Voir en outre HaEnsch 1997, p. 137 et MaURin, 2005, p. 10 qui rappelle que c'était déjà le « sentiment de Camille Jullian » : précisément, le raisonnement suivi ici relève de l'impression non de la démonstration.

18 HaEnsch, 1997, p. 26 et p. 859 (indices). Caput provinciae est présent dans le Bel. Hisp., 31, et dans quelques rares textes plus tardifs. Tacite l'utilise parfois suivi du nom de la province. Le vocable sedes privilégie la résidence habituelle du magistrat ; caput, de tonalité plinienne (supra n. 10), s'emploie pour des villes placées au premier rang de leur région, suggérant une supériorité qui n'est pas nécessairement de caractère administratif ou territorial : voir caput Celtiberiae accolé à Segobriga (N. H., III, 25).

19 CIL, XIII, 1129 (Limonum et Lemonum sont tous deux attestés suivant un phénomène linguistique fréquent dans les toponymes d'époque romaine dans les Gaules et ailleurs : voir Redones et Riedones) : il s'agit d'un hommage public à Claudia Varenilla, épouse de M. Censorius Paullus qui est parfois identifié au consul suffect de 160 p. C. : voir AlföLDY, 1977, p. 174 fondé sur les « Fastes d'Ostie ». Une révision du document en 1997 (Bargagli-Grosso, Itinerari Ostiensi VIII, 1997, p. 50-51), conduit à proposer Caesorius et non plus Cesorius pour Ce(n)sorius Paulus avec un seul L. : la photographie ne confirme pas cette nouvelle hypothèse en l'absence de trace de A. Le formulaire épigraphique semble orienter vers le $\mathrm{II}^{\mathrm{e}}$ siècle et est difficilement postérieur au début du III ${ }^{\mathrm{e}}$.

Conimbriga, 49 (2010) 97-118 
élever une statue honorifique à un gouverneur ${ }^{20}$. En revanche, il n'est probablement pas négligeable de rencontrer à Bordeaux l'inscription d'un decurialis lictor originaire de Rome, décédé sur les bords de la Garonne et enterré par les soins de son affranchi et héritier ${ }^{21}$. Une fois encore l'argument est un indice et non une preuve en raison de la mobilité des gouverneurs et de leurs légats.

L'urbanisation et la monumentalisation de la ville, les conjonctures économiques que révéleraient les vestiges archéologiques sont enfin invoqués, de même que la circulation monétaire ou les hommages aux empereurs notamment julio-claudiens ${ }^{22}$. D'un strict point de vue méthodologique, il n'est pas difficile de constater que les arguments qui sont invoqués ne peuvent convenir. Si Narbonne n'avait pas donné son nom à la province ${ }^{23}$ et n'était pas dûment répertoriée dans les sources comme caput Galliae narbonensis, Arles aurait du point de vue archéologique au moins autant sinon plus de titres qu'elle à être la capitale. Si un arc de ville suffisait, Orange aurait aussi un mot à dire et Vienne par sa parure ne serait pas en reste. Le patrimoine archéologique d'Autun et son nom pourraient, dans cette perspective, suggérer une capitale. Reims ne surpassait pas Trêves en matière d'urbanisme et ne bénéficiait pas des atouts géographiques de la cité des Belges. Dans la péninsule Ibérique, Hispalis n'avait rien à envier sur tous ces plans à Cordoue. Les hommages impériaux abondent dans toutes les cités importantes ou dynamiques sous l'Empire indépendamment de leur rang provincial et de leur taille urbaine. Le financement de l'amphithéâtre de Condate par le descendant de la puissante famille des Iulii de Saintes, ayant aussi manifesté activement son attachement à la domus Augusta à Saintes même ${ }^{24}$, nous informe davantage sur les ambitions de ces notables que sur le statut administratif de la cité. Il en va de même pour les arguments d'ordre économique tirés des vestiges supposés refléter une prospérité expliquant un choix du pouvoir romain, même

20 CIL, XIII, 1803 ; voir HAENSCH, 1997, p. 464-465.

21 ILS, 1906 ; HAENSCH, 1997, p. 464. Le texte est incomplet au début, d'où l'anonymat du personnage.

22 TRAnOY, 2004, p. 234- 236 plus particulièrement ; MAURIN, 2005, p. 11-13.

23 Narbonensis, Tarraconensis pour désigner la province indiquent sans ambiguïté que ces deux cités étaient les résidences des gouverneurs malgré le septicisme de $\mathrm{R}$. Haensch, peu compréhensible.

2424 Voir ILTG, 217 et le dossier dans Tranoy, 2004, p. 231-235 et Maurin, 2005, p. 9 .

Conimbriga, 49 (2010) 97-118 
si les capitales provinciales ont bénéficié assurément de leur position en termes de croissance urbaine postérieure ${ }^{25}$.

Les faiblesses du dossier que je viens de rappeler sont présentes à l'esprit de tous ceux qui l'ont rassemblé26. Les difficultés des recherches sur le terrain constituent un obstacle trompeur à l'heure des conclusions. Une étude récente de M. Navarro Caballero a montré que des arguments utilisés pour Saintes valaient aussi pour Burdigala moins aisée à étudier en raison d'un essor urbain contemporain plus défavorable à la conservation des vestiges et au travail de terrain ${ }^{27}$. La mise en évidence minutieuse d'hommages julio-claudiens dans le secteur du mont Judaïque à l'époque de Tibère, fondée sur l'histoire des découvertes et sur les restes des statues inventoriés, atteste que les conclusions archéologiques sont étroitement tributaires de l'intensité et de la qualité des recherches dont les limites ne peuvent se confondre avec l'inexistence de telle ou telle réalité antique. Pour proposer une solution qui convienne ou convainque, il est jugé de bonne méthode de recourir à toutes les formes possibles d'argumentation. Pourtant, tous les indices n'ont pas la même valeur - on vient de le voir - et manquent de solidité à l'examen. Il en est ainsi des routes et du texte de Strabon - nous y reviendrons dans un instant - sur le réseau d'Agrippa ${ }^{28}$ : rien n'indique dans ce passage que le réseau fut conçu en fonction de capitales administratives et ce sont plutôt les extrémités géographiques d'ouest en est (l'Aquitaine, le Rhin, l'Océan, Marseille) qui sont privilégiées. De même que c'est Marseille et non Narbonne qui est mentionnée, de même les Santons sont-ils cités suivis de l'Aquitaine, ce qui modifie la portée administrative et politique du propos, absente au profit de la géographie. Lugdunum elle-même n'est présentée que comme «le milieu du territoire », ce qui manque de précision et de rigueur et inclut la Narbonnaise ${ }^{29}$.

25 ÉTIENNE, 1962, p. 82-85 et 1995, p. 179 : Saintes subirait en revanche une perte de vitesse progressive au Haut-Empire. HAENSCH, 1997, p. 137-138 à l'aide surtout de Étienne, 1962.

26 Haensch, 1997, p. 135 ; Maurin, 2005, p. 10.

27 NAVARro, 2008, p. 197-229.

28 Strabon, IV, 6, 11.

29 Thollard, 2009, p. 120-121. F. Lasserre traduit sans motif par « le centre de la Celtique ", explicitant une formulation qui reste vague chez Strabon (voir infra n. 35).

Conimbriga, 49 (2010) 97-118 
Comment essayer, malgré tout, d'éclairer une question aussi peu intéressante pour les Anciens ? Ce manque d'intérêt n'est-il pas lui-même une source d'information?

\section{Burdigala siège du gouvernement provincial}

Robert Étienne, à la suite de Louis Maurin, avait retenu une solution ingénieuse consistant, faute de données autorisant une solution tranchée, à déplacer le siège de la capitale de Saintes à Poitiers au $\mathrm{II}^{\mathrm{e}}$ siècle puis à Bordeaux au $\mathrm{III}^{\mathrm{e}}$ siècle $^{30}$. Il s'agissait d'un compromis, ce qui en matière de recherche est rarement satisfaisant. R. Haensch a d'ailleurs rejeté cette solution qui constituerait un cas d'espèce dans l'ensemble de la pratique administrative romaine et, peut-on ajouter, n'aurait aucune justification claire et décisive ${ }^{31}$. L'idée que l'inscription CIL, XIII, 1697, érigée à Lyon, honorant un curateur des Bituriges Vivisques d'origine pictave induirait une subordination de Bordeaux par rapport à Poitiers est pour le moins surprenante ${ }^{32}$. Le curateur n'avait en aucun cas autorité sur la cité qu'il contrôlait. Sa mission temporaire, effectuée à la demande de l'ordo local, était de nature technique et financière et ne faisait pas du titulaire un magistrat ${ }^{33}$. Il faut en conséquence choisir un site indépendamment de tout préjugé le plus souvent lié à des données contemporaines parfaitement étrangères aux critères romains sur lesquels nous aurons à revenir.

Outre les lacunes des sources, l'histoire administrative de la province d'Aquitaine souffre d'une deuxième limitation. Les administrations

30 Voir le résumé et la bibliographie dans HAENSCH, 1997, p. 135-136.

31 HAENSCH, 1997, p. 136.

32 RACINES, 1992, p. 28-30.

33 JACQUES, 1990, p. 178, n 101, commente brièvement ce texte. F. Jacques a par ailleurs, à plusieurs reprises, insisté sur la déformation que constitue le fait d'interpréter la fonction de curateur de cité au Haut-Empire comme le témoignage d'un autoritarisme croissant du pouvoir central visant à mettre fin à l'autonomie des cités. L'intervention d'un curateur se faisait avec l'accord impérial sur sollicitation du gouverneur. Une cité ne pouvait pas, dans l'Empire romain, avoir autorité sur une autre cité sans une décision expresse du pouvoir (contributio/attributio): L. Lentulius Censorinus, pictave d'origine, n'était pas pour autant en la circonstance un représentant mandaté de sa cité. C'est le concile des Gaules qui a fait élever la statue parce que Censorinus avait revêtu la charge d'inquisitor pour le compte de l'assemblée des Trois Gaules au Confluent.

Conimbriga, 49 (2010) 97-118 
financières et les institutions religieuses de la province n'étaient pas circonscrites au ressort provincial mais relevaient de Lyon et partageaient parfois la fonction avec la Narbonnaise. Cette situation a eu pour effet d'enlever des indices épigraphiques, présents dans d'autres secteurs provinciaux, à la capitale provinciale comme l'a déjà remarqué $\mathrm{R}$. Haensch. Le raisonnement le plus efficace et le plus proche des faits est assurément celui que R. Haensch a suivi tout en estimant, à tort, que le texte d'Eutrope n'était pas fiable sur ce point : Burdigala capitale au $\mathrm{III}^{\mathrm{e}}$ et au $\mathrm{IV}^{\mathrm{e}}$ siècle était certainement la capitale originelle. On sait par Pline l'Ancien que Burdigala, à l'instar des Santons, était une cité libre, ce qui n'était pas le cas des Pictons ${ }^{34}$. Le statut et sa place hiérarchique n'étaient pas des éléments entrant nécessairement en ligne de compte même si les colonies étaient, sur ce plan, privilégiées dans les provinces occidentales à l'aune des circonstances historiques ayant présidé à la phase d'intégration politique. Mogontiacum (Mayence), Londinium (Londres) n'avaient pas ce statut mais abritèrent la résidence du gouverneur provincial. En Aquitaine, on ne connait pas de colonie ni de municipe et il est logique que la capitale n'ait pas autant qu'on le sache été promue à un rang privilégié, celui de municipe n'étant de toute façon guère vraisemblable en Gaule occidentale où il n'est nulle part attesté.

L'administration, le droit, les statuts civiques n'offrent pas de solution solidement étayée. La lecture du livre de R. Haensch, par son érudition et l'examen serré des sources semble décourager les meilleures volontés. Pourtant, il apparaît que l'auteur ne tire pas, dans ses raisonnements, tout le parti souhaitable de la géographie qu'elle soit celle des Anciens ou celle que nous connaissons aujourd'hui. Il semble même méfiant à son égard en raison d'un usage déterministe encore courant, ce qui néglige le fait qu'en matière de stratégie et d'administration le regard géographique entrait de façon non géographique dans les critères élaborés par le pouvoir. L'attention portée au relief, aux données naturelles favorables à la circulation et aux déplacements ou influant sur les campagnes militaires allait de pair avec l'exercice du contrôle des populations provinciales, ce qui n'implique aucune règle ni détermination exclusive. Les responsables administratifs ou les autorités locales ne pouvaient pas ne pas en tenir compte concrètement

34 Pline L'Ancien, N. H., IV, 17, 108 (voir aussi supra n. 6).

Conimbriga, 49 (2010) 97-118 
et Strabon le reflète parfaitement en insistant sur les cours d'eau, les obstacles montagneux et les mers.

Le fameux texte relatif au réseau routier d'Agrippa en est une illustration et en ce sens un bon point de départ pour une réflexion plus globale ${ }^{35}$ :

«Lugudunum se situe au milieu du territoire, à la façon d'une acropole, par la confluence des fleuves et en raison de sa proximité avec les autres parties. C'est pour cela qu'Agrippa en a fait le point de départ des routes : celle qui, à travers les monts Cemmènes, conduit jusqu'aux Santons et l'Aquitaine, celle qui se dirige vers le Rhin, une troisième vers l'océan du côté des Bellovaques et des Ambiens, une quatrième étant celle qui gagne la Narbonnaise et le littoral massaliotique... »

Il est notable que Strabon, qui est certainement fidèle à l'esprit d'Agrippa dans ce passage, retienne la situation favorable de Lugudunum à la fois carrefour et lieu visible (acropole) sans faire aucune allusion à une prééminence administrative de la colonie plancienne. Ni les Bellovaques, ni les Ambiens, ni Marseille ne correspondent non plus à cette catégorie. Les confins maritimes sont plus importants et indiquent qu'à l'odographie la géographie grecque ajoutait le périple ou navigation exploratoire et donc le rôle essentiel de la circumnavigation et des traversées entre terres relativement proches les unes des autres. L'eau et la terre se complétaient étroitement et se prolongeaient quand il s'agissait de choisir des itinéraires de déplacement. Il est ainsi légitime de conclure sans hésitation que la mention des Santons associée à l'Aquitaine n'a rien à voir avec le rang de capitale de la province auquel il eût peut-être été fait allusion si tel avait été le cas.

Le recours à une géométrie simplifiée en matière de cartographie des contrées et de leur situation relative, associée au rôle tenu par les principales artères fluviales ne peut pas être ignoré dans la recherche de la capitale de l'Aquitaine romaine ${ }^{36}$. Outre l'emplacement de Burdigala au milieu du quadrilatère aquitain selon une représentation bien mise

35 Strabon, IV, 6, 11.

36 Les déformations dues à l'absence d'instruments adaptés de calcul et de relevés n'ont pas d'importance. Ce qui compte est la construction d'un croquis fondé sur des principes semblables pour chaque région et dominé par des axes et des lignes directrices permettant de se repérer rapidement. Sur le terrain, ces « cartes » étaient moins utiles et utilisées que les itinéraires.

Conimbriga, 49 (2010) 97-118 
en évidence à partir des sources anciennes ${ }^{37}$, le site bordelais sur les rives de la Garonne à proximité de l'estuaire débouchant sur l'océan et touché par le phénomène des marées et la position de la ville à la jointure de l'Aquitaine césarienne et de l'Aquitaine augustéenne sont des éléments décisifs à suivre les pratiques et les conceptions romaines, sans oublier l'existence de l'isthme aquitain reliant Narbonne à Toulouse et Bordeaux, dont dès avant César les Romains étaient parfaitement conscients $^{38}$. Un inventaire des capitales des provinces occidentales montre que des normes souhaitables avaient été retenues pour leur désignation ou élection chaque fois que cela était possible : Carthage, Césarée de Maurétanie, Tingis, Tarragone, Narbonne, Cemenelum, Cordoue, Mérida, Lyon, Londres, Cologne, Mayence étaient des villes portuaires ou maritimes ou fluviales ou les deux à la fois. Le cas de Cordoue, préférée à Hispalis ou Gadès, peut paraître une anomalie. Comme pour Tarragone, l'histoire des guerres péninsulaires a influé sur le choix dans la mesure où une habitude ancienne avait fait de Cordoue une résidence habituelle des imperatores et proconsuls ${ }^{39}$. Grâce au Baetis navigable, la route de Rome était ouverte et par terre et par mer. Dans le cas des Gaules, de soumission récente, les conditions du choix d'une capitale provinciale n'étaient pas influencées par un passé plus ou moins ancien de présence romaine ou de traditions gauloises contraignantes.

Ce n'est pas en fonction d'équilibres gaulois hypothétiques ni d'une unité des Trois Gaules, inexistante, que peut résider la solution. Lyon n'a jamais été à proprement parler la capitale des Trois Gaules et chaque gouvernement provincial était indépendant des autres, ne devant des comptes qu'à Rome. Le choix de Burdigala, dicté par une certaine géographie, prend donc tout son sens par rapport à Rome et aux relations constantes nécessaires entre l'Vrbs et les provinces. De ce point de vue, il est incontestable que Bordeaux était mieux placée que Saintes et Poitiers qu'il se soit agi de navigation fluviale, de route terrestre ou de voyage maritime. En revanche, l'accessibilité de la capitale à partir des différents points de la province n'était pas vraiment prise

37 Voir ThOLLARD, 2009, p. 120-121 et les cartes élaborées par C. Goudineau et P. Thollard.

38 Voir parmi d'autres, le Catalogue collectif de l'exposition intitulée La voie de Rome entre Méditerranée et Atlantique, Ausonius, 2008, en particulier page 29-31. Aussi, Le Roux, 2010, p. 122-125.

39 Le Roux, 2010, p. 62.

Conimbriga, 49 (2010) 97-118 
en considération sachant que le caractère central n'était pas recherché par principe et ne s'observe que rarement. Ces modes de raisonnement l'emportèrent à l'époque augustéenne, date d'institution des capitales des Gaules, sur tous les autres. Les archives et les instruments de gouvernement ordinaires n'avaient pas le poids qui leur est conféré aujourd'hui et aucun système centralisé n'existait à l'échelle provinciale. Comme Hadrien ou d'autres empereurs, les gouverneurs se déplaçaient avec leurs auxiliaires et leur documentation indispensable lorsqu'il le fallait. Autant qu'il soit possible de le savoir, la tradition n'a pas retenu de conflit, de sollicitation ou de compétition entre des cités pour obtenir le statut de capitale d'une province, même en Orient où les revendications des Grecs portaient sur la néocorie et le rang de (première) métropole indépendant de celui de caput provinciae : ce faisant, les cités grecques perpétuaient une tradition politique grecque que Rome se contentait d'observer et maintenir dans des limites acceptables ${ }^{40}$. Comme l'a en outre observé R. Haensch ${ }^{41}$, il n'y avait pas de lien obligatoire entre le concilium et la capitale. De même, à Lyon, le Confluent était extérieur à la colonie-capitale et échappait à sa tutelle.

Une remise à plat de la réflexion propose sans ambiguité Burdigala comme caput provinciae dès l'époque d'Auguste et pour la durée globale de la province augustéenne. Rien n'oriente vraiment vers Saintes ni vers Poitiers ${ }^{42}$. L'histoire administrative doit être dissociée d'autres paramètres qui ne jouèrent pas directement un rôle dans la décision de faire de telle ou telle ville la capitale d'une province. La recherche de l'efficacité dans le contexte de pratiques de gouvernement et d'administration différents de ceux qui nous sont familiers intervenait en premier lieu. La capitale n'avait pas dans la province le rayonnement que lui confèra par la suite un état centralisé et bien d'autres villes-cités rivalisaient avec elle en ornementation, en population et en prospérité. La présence du gouverneur ne déboucha que très rarement sur des générosités et embellissements locaux de son fait ${ }^{43}$. Ce n'est sans doute

40 Voir Heller, 2006, p. 372-375.

41 HaEnsch, 1997, p. 851 (indices, s. v. concilium) et p. 63.

42 Voir aussi TASSAUX, 2003, p. 57-60.

43 HaENSCH, 1997, p. 377-389. Les initiatives des magistrats sont cependant plus nombreuses à partir du $\mathrm{III}^{\mathrm{e}}$ siècle, semble-t-il, et impliquent l'empereur. Il n'y eut toutefois jamais de volonté de donner à la capitale une parure monumentale exceptionnelle, différente de celle des autres cités de la province.

Conimbriga, 49 (2010) 97-118 
pas par hasard que les familles dynamiques et soucieuses d'ascension sociale ne provenaient que rarement des capitales administratives ${ }^{44}$. Une réalité propre à l'Empire romain pesait sur le statut et la place des capitales, la dissociation entre les tâches administratives et la puissance politique. Le gouverneur ambitieux et soucieux de sa carrière regardait vers Rome plus que vers sa province. En revanche, les élites étaient attirées par la résidence du magistrat qu'elles pouvaient solliciter ou obliger commodément selon les circonstances. Le gouverneur était en partie itinérant. Avec le temps, l'institution de bureaux et de circuits adminisitratifs stables ancra durablement une ville dans sa fonction de capitale, mais les provinces ni les capitales ne furent jamais conçues comme immuables et définitives ${ }^{45}$.

\section{L'Aquitaine et la provincia romaine}

Plus qu'à l'essor des sociétés et des villes provinciales, plus encore qu'à la « romanisation » trop usée à force d'un emploi historiographique mécanique, la question d'une capitale provinciale se rapporte essentiellement à l'exercice même de l'autorité dans le cadre d'une «provincia». Le sens de cette institution, les tâches qu'impliquait son fonctionnement éclairent au premier chef la notion même de capitale. De ce point de vue, l'Aquitaine augustéenne ou flavienne n'était pas une province particulièrement enviable dans la hiérarchie provinciale bien que classée dans le lot des provinces du César, les plus prestigieuses à rang égal comparées aux provinces publiques ou proconsulaires et davantage encore aux provinces dites " équestres ». Sauf durant une (ou deux ?) brève(s) période(s), la province d'Aquitaine appartint à la catégorie des secteurs « inermes » et n'abrita jamais aux deux premiers siècles d'unités autres qu'auxiliaires. Tacite en apporte une confirmation en évoquant l'activité d'Agricola dans la province :

«Agricola naturali prudentia, quamvis inter togatos, facile iusteque agebat. Jam vero tempora curarum remissionumque divisa : ubi conventus ac iudicia poscerent, gravis, intentus, severus et saepius misericors ; ubi

44 Voir par exemple BURNAND, 1984, p. 424-429.

45 Il suffit de mentionner les nombreuses créations et divisions au cours du Haut-Empire sans parler de la refonte territoriale de Dioclétien.

Conimbriga, 49 (2010) 97-118 
officio satisfactum, nulla ultra potestatis persona : tristitiam et arrogantiam et avaritiam exuerat ; nec illi, quod est rarissimum, aut facilitas auctoritatem aut severitas amorem diminuit. Integritatem atque abstinentiam in tanto viro referre injuria virtutum fuerit. Ne famam quidem, cui saepe etiam boni indulgent, ostentanda virtute aut per artem quaesivit : procul ab aemulatione adversus collegas, procul a contentione adversus procuratores, et vincere inglorium et atteri sordidum arbitrabatur. Minus triennium in ea legatione detentus ac statim ad spem consulatus revocatus est... ${ }^{46} »$.

Le texte est une louange du beau-père et des vertus révélatrices d'un excellent gouverneur. En filigrane, les tâches d'un légat d'Aquitaine et leur contexte provincial n'en sont pas moins présents. La gloire de Galba avant lui ${ }^{47}$ et d'Agricola ne doivent pas déformer les hiérarchies ni faire croire que l'Aquitaine était particulièrement recherchée ou prestigieuse. Trois ans semblent constituer la durée habituelle de la fonction sous Vespasien. Les provinciaux sont définis comme togati, ce qui signifie qu'ils vivent en cité et conformément aux règles que cela suppose, à l'opposé des comportements des peuples belliqueux. Les procurateurs mentionnés suggèrent que le légat devait intervenir en cas de difficultés dans la perception de l'impôt et des taxes mais conventus ac iudicia constituent l'essentiel en l'absence d'activités militaires. Conventus, au pluriel, accolé de manière redondante à iudicia, suivant une figure de style bien connue, évoque les assises judiciaires et non une quelconque

46 TACITE, Agric., IX : «Agricola, grâce à sa clairvoyance naturelle, quoiqu'il ê̂t affaire à des togati, accomplissait sa tâche avec bonne humeur et équité. Il avait d'ailleurs divisé son temps entre travail et détente : là où les assises et les procès l'exigeaient, il se montrait grave, attentif, sévère et plus souvent compatissant; après l'accomplissement de ses obligations, plus rien n'évoquait en lui le masque du pouvoir : plus de visage fermé ni d'air hautain ni d'âpreté; chez lui, ce qui est très rare, l'affabilité n'enleva rien à son autorité ni la sévérité à l'amour d'autrui. Parler chez un si grand homme de son caractère intègre ou de son désintéressement serait faire injure à ses vertus. La renommée elle-même, envers laquelle même les gens honnêtes font preuve de complaisance, il ne la rechercha point en étalant son courage ou à force d'habileté : il éloigna toute rivalité envers ses collègues et tout conflit avec les procurateurs; il estimait qu'il n'y avait aucune gloire à vaincre, mais de la honte à être écrasé. Occupé depuis moins de trois ans dans cette fonction de légat, il fut rappelé avec aussitôt la promesse du consulat...».

47 Galba fut gouverneur sous Tibère, vers 31-32 (Suétone, Galba, 6) à une époque où les hiérarchies et les carrières n'étaient pas aussi structurées qu'à partir de Vespasien : Galba et Agricola étaient tous deux patriciens au moment de leur gouvernement.

Conimbriga, 49 (2010) 97-118 
assemblée ou encore moins un conventus de citoyens romains. R. Haensch interprète ce passage comme une illustration d'une idée qui traverse son livre ${ }^{48}$ : toutes les provinces ont connu les conventus judiciaires qui ne se limitent donc pas à ceux que Pline fait connaître directement en Hispania ou en Dalmatie ni aux diocèses d'Asie.

De quoi pouvait-il s'agir ? Chaque année, les légats d'Aquitaine devaient effectuer une tournée dans leur province au titre de l'activité judiciaire qui ne relevait pas de la justice locale. Installé à Burdigala, le légat gouverneur ne se contentait pas de siéger dans son seul prétoire bordelais. L'insistance de Tacite sur le fait que les gouvernés étaient des togati rappelle que l'activité judiciaire était d'autant plus développée que les règles juridiques familières aux Romains étaient en cause. Rien ne signale jusqu'à présent dans la documentation, notamment épigraphique, l'existence d'un légat assistant le gouverneur d'une province prétorienne assez peu étendue ${ }^{49}$, ce qui était, semble-t-il, la situation ordinaire des légations impériales sans légion comme le confirment la Lusitanie ou la Gaule Lyonnaise ${ }^{50}$. Dans des circonstances exceptionnelles, le recours à un auxiliaire était toujours possible cependant notamment en période de recensement.

Le circuit annuel existait dès le départ mais était certainement déterminé par l'importance des affaires à traiter, ce qui rendrait en partie compte de l'absence de mention précoce, disons julio-claudienne, de circonscriptions judiciaires dûment officialisées dans la formule provinciale. La péninsule Ibérique dans laquelle Pline l'Ancien, qui y fut procurateur, inventorie 14 conventus pour l'ensemble des trois provinces avait fait l'objet de la mise en place de pratiques administratives depuis deux siècles, source d'expériences codifiées peu à peu au cours du temps. Ce ne pouvait pas être le cas des Gaules bien que, logiquement, Rome n'ait eu aucune raison de ne pas y appliquer des recettes identiques. Il est donc probable qu'il faille admettre l'émergence progressive de centres urbains dans lesquels les légats prirent l'habitude

48 HaEnsch, 1997, p. 18-36 et p. 136 pour l'Aquitaine.

49 L'Aquitaine augustéenne devait mesurer $165000 \mathrm{~km}^{2}$ environ (RACINES, 1992, p. 28), soit un peu plus de la la moitié de l'Espagne citérieure mais plus compacte qu'elle et moins montagneuse en dehors de certaines régions du Massif central. La circulation n'y soulevait pas de difficultés particulières.

50 Voir également Lassère, 2005, p. 654. Sur la carrière d'Agricola, Ibid., p. 647-648 .

Conimbriga, 49 (2010) 97-118 
de convoquer leurs assises au cours de leur tournée. Force est de dire que, sur ce plan aussi, aucun indice épigraphique ou autre ne milite en faveur de Saintes, trop proche de Bordeaux et de Poitiers. Limonum possède en revanche deux témoignages qui peuvent rendre compte du choix de siège de conventus : l'inscription de Claudia Varenilla déjà mentionnée et celle de M. Sedatius Severianus, honoré comme patron de cité dans ce qui était sa ville d'origine, Poitiers ${ }^{51}$. Le décès de la première s'expliquera sans doute, on l'a vu, par un séjour de son mari en qualité de gouverneur, ce qui peut correspondre à un déplacement pour la tenue du conventus annuel. L'ascension des Sedatii et l'hommage au patron de cité se comprend mieux si Poitiers facilitait régulièrement un contact des notables avec le gouverneur, sachant qu'une capitale provinciale était paradoxalement moins favorisée parfois que les autres cités pour l'établissement de relations amicales.

Une autre candidate à un siège de conventus dans la province pourrait être Lugdunum Convenarum. A. Chastagnol a mis en doute à juste titre l'existence dans la cité des Convènes d'un concile du culte impérial propre aux « neuf peuples $»^{52}$. La présence d'un concilium ne serait de toute façon pas un argument suffisant, nous l'avons vu. L'absence d'indices épigraphiques suggérant la présence du gouverneur invite à écarter cette candidature. En outre, dans le contexte de l'Aquitaine préaugustéenne, c'est Eauze, la cité des Elusates, qui tiendrait la corde comme siège du conventus : son rang de capitale de la Novempopulanie dans l'Antiquité tardive ${ }^{53}$ prend davantage de consistance si la cité avait accueilli périodiquement les assises judiciaires aux époques antérieures. En l'absence de documents probants ou suggérant une possible interprétation en ce sens, il serait hasardeux d'attribuer à tout prix aux régions orientales un siège à choisir entre Cahors, Limoges ${ }^{54}$

51 Voir ÉTIENNE, 1995, p. 229-234 avec la bibliographie. L'abréviation du gentilice prouve que le personnage était renommé non qu'il était originaire de Poitiers, ceci n'empêchant pas cela comme l'indique le choix de la cité pour l'hommage. Récemment HIERNARD, 2007, p. 231-264, est revenu très longuement sur ce document avec un bonheur inégal : il en résulte cependant, outre une nouvelle restitution du support et du texte, que la cité dédicante n'est pas nécessairement aquitaine et encore moins cadurque, ce qui avait déjà suscité des critiques diverses.

52 Hypothèse de Maurin, 1978, p. 200 : Chastagnol, 1995, p. 35.

53 RACINES, 1992, p. 33.

54 Voir aussi supra n. 20 : les inscriptions de Lyon (CIL, XIII, 1803) et de Rome (CIL, VI, 1568) en l'honneur d'un gouverneur par les Lémovices et les Cadurques

Conimbriga, 49 (2010) 97-118 
ou Bourges. Poitiers et Bordeaux faisaient sans doute l'affaire mais Bourges, chef-lieu des Bituriges Cubes et capitale de l'Aquitaine première après Dioclétien aurait ainsi aussi un titre à avoir accueilli pendant longtemps les assises judiciaires des gouverneurs. Il serait évidemment plus simple de disposer d'un document comparable au texte de Pline fondé sur les formules augustéennes ou de peu postérieures. Poitiers, Bordeaux, Eauze et Bourges furent vraisemblablement des sièges des conventus aquitains. Il est possible qu'en certaines circonstances, à l'image de Calagurris dans la péninsule Ibérique, telle ou telle ville ait accueilli irrégulièrement ou exceptionnellement le gouverneur durant sa tournée ${ }^{55}$. L'existence de circonscriptions stables serait logique mais, à l'échelle des cités gauloises, leur définition précise n'était peut-être pas aussi nécessaire et durable que dans d'autres provinces de l'Empire ${ }^{56}$. Une évolution en ce domaine est admissible à la différence de ce qui se passait pour la capitale provinciale.

Contrairement à ce qui était envisageable, les hésitations sur la capitale de la province ne sont pas compensées par l'identification des capitales de conventus sauf dans le cas de Poitiers. Force est d'admettre que les pratiques imposées par l'expérience et des critères jamais énoncés dans les sources induisaient des choix qu'il serait vain de vouloir rendre logiques et rationnels selon des normes supposées objectives et pérennes. Une province romaine était une construction complexe reposant sur des conceptions pragmatiques en termes de contrôle des populations. Elle n'était pas l'expression d'un état souverain libre de son organisation et centralisé. Les villes et cités importantes n'étaient pas, en tout cas dans les provinces occidentales de Rome, en compétition pour le premier rang et pour remettre sans cesse en cause une hiérarchie aussi souple que peu contraignante. Le gouverneur n'était pas le monarque d'un territoire dont il pouvait disposer souverainement. Burdigala, capitale ou non, valut par le dynamisme de ses élites et par la beauté de sa parure monumentale à l'instar des autres villes importantes de la province : Poitiers, Bourges, Limoges, Saintes, Cahors, Périgueux, Elusa, Lugdunum Convenarum et

relèvent des rapports de clientèle et d'amitié ou de patronage et ne sont pas des preuves que ces cités étaient des sièges d'assises judiciaires.

55 Le Roux, 2010a, p. 133-134. La présence de sources thermales ou d'eaux jugées salutifères justifiait peut-être un choix temporaire et jamais renouvelé ensuite.

56 Ce n'est pas une question de chronologie mais d'évolution fluctuante du volume des affaires à instruire et d'inégalités régionales au sein même de l'Aquitaine.

Conimbriga, 49 (2010) 97-118 
d'autres encore que les réalités d'aujourd'hui feraient croire modestes ou sans importance. La capitale n'était pas un repère indispensable en dehors des préocccupations administratives, ce qui éclaire le désintérêt relatif des sources pour ces fonctions.

Le fait qu'une capitale provinciale de l'Empire romain n'ait pas été un centre politique mais le lieu de résidence du gouverneur permet de dégager l'horizon d'une histoire administrative ni moins ni plus intéressante qu'au départ de la recherche effectuée ici. Le rang de capitale s'en trouve en quelque sorte dédramatisé et Burdigala n'a pas pour autant gagné en richesse historique qu'une perspective trop « moderne » et egocentrée avait voulu lui conférer. C'est l'histoire postérieure longue qui a construit au cas par cas la ville d'aujourd'hui. La part romaine a assuré sa durée mais ce sont, sous l'Empire, sa population et ses élites qui ont fait le prestige et la renommée d'une cité qui partageait ce désir de gloire avec toutes les autres villes de la province et du monde romain. Une province telle que l'Aquitaine - et les autres - était un lieu de contrôle des populations locales soit par la loi soit par la force. Dans des contextes pacifiés, le gouverneur effectuait des déplacements fréquents destinés à inspecter les régions traversées et à s'informer autant qu'à assurer l'exercice de la justice dans le cadre des conventus annuels. Ces données confirment que l'administration romaine était avant tout organisée autour des activités concrètes en fonction d'une priorité aux relations avec chaque communauté qui, aux yeux du gouvernement, étaient toutes peu ou prou équivalentes car elles étaient susceptibles à un moment ou un autre de soulever des difficultés, ce qui ne veut pas dire que cela arrivait systématiquement ni que les réactions étaient créatrices de risques et de dangers. Enfin, sans nier que les questions de représentation du pouvoir aient pu se greffer épisodiquement sur la visibilité d'une capitale, elles ne furent pas l'essentiel, pas davantage que la dimension économique ou culturelle. L'empire n'avait qu'un seul centre exprimant sa puissance et sa prospérité: l'Vrbs. Quand l'empereur n’y résidait pas Rome se trouvait là où était l'empereur.

Conimbriga, 49 (2010) 97-118 


\section{BIBLIOGRAPHIE}

AlföLDY, 1977 : Géza Alföldy, Konsulat und Senatorenstand unter den Antoninen. Prosopographische Untersuchungen zur senatorischen Führungsschicht, Rudolf Habelt, Bonn.

BAts, 2007 : Droit latin, adtributio et contributio. Strabon, Pline, Nîmes et les Volques Arécomiques, $M E F R A, 119,1$, p. 51-62.

BuRNAND, 1984 : Yves Burnand, Senatores romani ex provinciis Galliarum, dans EOS, II, Tituli 5, Rome, p. 387-437.

Chastagnol, 1995 : André Chastagnol, La Gaule romaine et le droit latin. Recherches sur l'histoire administrative et sur la romanisation des habitants, Scripta varia 3, CEROR no 14 , Lyon.

ÉTIENNE, 1962 : Robert Étienne, Bordeaux antique (avec la collaboration de P. Barrère), Bordeaux.

ÉTIENNE, 1995 : Robert Étienne, En passant par l'Aquitaine..., Recueil d'articles de Robert Étienne, Recherches et travaux d'histoire sur le Sud-Ouest de la France, Bordeaux.

HaEnsch, 1997 : Rudolf Haensch, Capita provinciarum. Statthaltersitze und Provinzialverwaltung in der römischen Kaiserzeit, Kölner Forschungen 7, Philip von Zabern, Mayence.

Heller 2006 : Anna Heller, "Les bêtises des Grecs ». Conflits et rivalités entre cités d'Asie et de Bithynie à l'époque romaine (129 a.C.-235 p. C.), Scripta antiqua 17, Ausonius éditions, Bordeaux.

HieRnARD, 2007 : Jean Hiernard (avec la collab. de Jacques Seigne), L'inscription de Poitiers en l'honneur du sénateur Severianus. Trente ans après, Revue historique $d u$ Centre-Ouest (Soc.Antiq.Ouest), VI, 2007, p. 231-264.

JACQUES, 1990 : François Jacques, Les Cités de l'Occident romain du ${ }^{\mathrm{er}}$ siècle avant J.-C. au VI siècle après J.-C., La roue à livres : Documents, Les Belles Lettres, Paris.

LASSÈRE, 2005 : Jean-Marie Lassère, Manuel d'épigraphie romaine, Antiquité / Synthèses 2 vol., Picard, Paris.

Le Roux, 1982 : Patrick Le Roux, L'armée romaine et l'organisation des provinces ibériques d'Auguste à l'invasion de 409, Diffusion de Boccard, Paris.

Le Roux, 2010: Patrick Le Roux, Sur Toulouse et les Toulousains sous l'Empire romain, dans Ab Aquitania in Hispaniam. Mélanges d'histoire et d'archéologie offerts à Pierre Sillières, Pallas, 82, p. 121-137.

Le Roux, 2010a : Patrick Le Roux, La péninsule Ibérique aux époques romaines, fin $d u$ III siècle av. n.è. - début du VI siècle de n.è., Collection U, A. Colin, Paris.

MAURIN, 1978 : Louis Maurin, Saintes antique des origines à la fin du VIe siècle après Jésus-Christ, Musée archéologique, Saintes.

MAURIN, 2005 : Louis Maurin, Saintes, lieu du colloque, dans L'Aquitaine et l'Hispanie septentrionale à l'époque julio-claudienne. Organisation et exploitation des espaces provinciaux, IV colloque Aquitania, Saintes, 11-13 septembre 2003, Aquitania, Supplément 13, Bordeaux, p. 9-13.

Conimbriga, 49 (2010) 97-118 
NAVARro, 2008 : Milagros Navarro Caballero, Du nouveau sur Burdigala : les hommages à la famille julio-claudienne, dans D'Orient et d'Occident, Mélanges offerts à Pierre Aupert, textes réunis par Alain Bouet, Mémoires 19, Ausonius éditions, Bordeaux.

RACINES, 1992 : Les Racines de l'Aquitaine. Vingt siècles d'histoire d'une région vers 1000 avant J.-C.- vers 1000 après J.-C., sous la direction de L. Maurin, J.-P. Bost, J.-M. Roddaz, Université Michel de Montaigne - Bordeaux III, Bordeaux. TASSAUX 2003 : Francis Tassaux, Poitiers fut-elle réellement capitale de l'Aquitaine?, dans Mélanges offerts à Louis Maurin. Itinéraire de Saintes à Dougga, Textes réunis par Jean-Pierre Bost, Jean-Michel Roddaz, Francis Tassaux, Mémoires 9, Ausonius-Publications, Bordeaux, p. 57-60.

Thollard, 2009 : Patrick Thollard, La Gaule selon Strabon. Du texte à l'archéologie. Géographie, livre IV traduction et études, Bibliothèque d'Archéologie méditerranéenne et africaine - 2, Éditions Errance / Centre Camille Jullian, Aix-en-Provence.

Tranoy, 1984 : Alain Tranoy, Centralisme, décentralisation ou provincialisation ? L'exemple de deux régions atlantiques d'Auguste à Constantin, $R H, 272 / 2$, p. 273-294.

Tranoy, 2004 : Laurence Tranoy, Mediolanum Santonum, Saintes : de la fondation jusqu'à l'époque julio-claudienne, dans Simulacra Romae. Roma y las capitales provinciales del Occidente europeo, Estudios arqueológicos, reunión celebrada en Tarragona los dias 12, 13 y 14 de diciembre del 2002, Tarragone, p. 223-237.

Conimbriga, 49 (2010) 97-118 